\title{
The Therapeutic Potential of Epigenetic Modifications in Alzheimer's Disease
}

\author{
Enric Bufill ${ }^{1}$ • Roser Ribosa-Nogué2 • Rafael Blesa² \\ ${ }^{1}$ Neurology Department, Vic University Hospital, Vic, Spain; ${ }^{2}$ Memory Unit, Neurology \\ Department, Hospital de la Santa Creu i Sant Pau, Biomedical Research Institute Sant Pau, \\ Universitat Autònoma de Barcelona, Barcelona, Spain
}

Author for correspondence: Enric Bufill, Neurology Department, Vic University Hospital, Vic, Spain. Email: ebufill@telefonica.net

Doi: https://doi.org/10.36255/exonpublications.alzheimersdisease.2020.ch9

\begin{abstract}
Alzheimer's disease is characterized by the formation and deposit of abnormal peptides such as amyloid plaques and neurofibrillary tangles in the brain. Therapeutic strategies aimed at preventing the formation of such deposits have not been successful. Currently, there are no effective treatments for the disease. Since numerous epigenetic changes have been detected in Alzheimer's disease, treatments aimed at reversing these changes by intervening in DNA methylation, histone acetylation, and microRNA expression may constitute promising lines of research in the future. This chapter provides an overview of the epigenetic changes and the potential epigenetic therapies in Alzheimer's disease.
\end{abstract}

Keywords: Alzheimer's disease; DNA methyltransferase; epigenetic changes; histone acetylation; noncoding RNA

In: Alzheimer's Disease: Drug Discovery. Huang X (Editor). Exon Publications, Brisbane, Australia. ISBN: 978-0-6450017-0-9; Doi: https://doi.org/10.36255/exonpublications.alzheimersdisease.2020 Copyright: The Authors.

License: This open access article is licenced under Creative Commons Attribution-NonCommercial 4.0 International (CC BY-NC 4.0) https://creativecommons.org/licenses/by-nc/4.0/ 


\section{INTRODUCTION}

Alzheimer's disease (AD) is the most common cause of dementia. It usually occurs in people over 60 years of age and presents with progressive loss of memory and cognitive capacity, language disorders, inability to translate ideas into actions (ideomotor apraxia), impaired planning and judgment, apathy, depression, and, in later stages, psychosis with paranoid delusions. AD is characterized by the presence of abnormal peptide deposits in the brain. The most characteristic lesions are neuritic extracellular plaques of the amyloid $\beta(A \beta)$ peptide, which consists of 33-40 amino acids derived from the proteolysis of the transmembrane protein amyloid precursor protein or APP. These neuritic plaques contain a large number of distorted neuronal expansions, known as dystrophic neurites. Activated microglial cells are observed at their center.

Some evidence suggests that amyloid deposits may be neurotoxic and may cause neuronal dysfunction and even neuronal death. In the normal brain, APP is fragmented into functional segments by the $\alpha-, \beta$-, and $\gamma$-secretase enzymes. Occasionally, there is an increase in $\beta$ - and $\gamma$-secretase relative to $\alpha$-secretase, leading to the accumulation of peptides with 40 and 42 amino acids, known as amyloid $\beta 40\left(A \beta_{40}\right)$ and amyloid $\beta_{42}\left(A \beta_{42}\right)$. The $A \beta_{42}$ peptide appears to have greater neurotoxic properties. A $\beta$ oligomers, small aggregates of 2-12 peptides, appear to be especially toxic (1). Diffuse plaques, another kind of plaque, lack a dense center of amyloid and dystrophic neurites. Unlike neuritic plaques, they are not associated with either neuronal destruction or cognitive dysfunction (2).

Neurofibrillary tangles are twisted aggregates of abnormal intraneuronal fibers that have a helical structure, typically paired helical filaments, made up of hyperphosphorylated tau protein. The tau protein is involved in stabilizing microtubules, maintaining the integrity of the cytoskeleton and axoplasmic transport. Neurofibrillary tangles are found in the areas of association of the neocortex, hippocampus, limbic system, substantia nigra, raphe nuclei, locus coeruleus, and the nucleus basalis of Meynert (3). In AD, there is also a significant synaptic loss in certain areas of the neocortex and in the hippocampus, as well as the disappearance of dendritic spines.

$\mathrm{AD}$ occurs frequently in humans over 65 years of age. In those aged over 85 , the prevalence of AD ranges between 20 and $40 \%$ in developed countries (3). In 2010, the prevalence of $\mathrm{AD}$ in China among people aged between 85 and 89 was $18.54 \%$ (4). In 2006, the number of patients with AD was 26.6 million worldwide. In the United States, the prevalence of AD in people over 70 years of age is $9.51 \%$, and the incidence is 14.26 per 1000 person-years (4). However, AD is not an inevitable consequence of old age. A relatively high number of elderly people show neither cognitive decline nor lesions typical of AD with age. The causes of $\mathrm{AD}$ are still not well understood. In a small percentage of cases, $\mathrm{AD}$ can be attributed to mutations in genes located on chromosomes 1, 14, and 21 . These cases are usually of early onset and are transmitted in an autosomal dominant manner. Most $\mathrm{AD}$ cases appear to be caused by the interaction of multiple genetic and environmental factors that are not yet well understood (1).

Areas of association, phylogenetically more recent areas of the human brain, have simpler organization and greater immaturity in the adult than phylogenetically older primary areas. Thus, in the neurons belonging to the areas of 
association, myelination occurs very slowly and many neurons belonging to these areas remain incompletely myelinated-that is, immature even in adulthood. Poorly myelinated neurons are chronically subjected to high-energy turnover, which makes them more vulnerable to the influence of oxidative stress. There are, therefore, extensive cortical areas in the human brain that remain structurally immature throughout life (5).

Various studies show that there has been an increase in the expression of genes related to aerobic metabolism and, more importantly, to synaptic plasticity and activity in the human cerebral cortex relative to nonhuman primate brains $(6,7)$. Learning and memory take place through the formation of new synapses and remodeling of preexisting synapses, suggesting that the increase in the expression of genes related to these functions has occurred in humans, as well as the selection of genes that encode proteins capable of increasing neuroplasticity. The apolipoproteins E (ApoEs) are proteins of 299 amino acids synthesized in the astrocytes of the central nervous system. They influence the transport and reuptake of cholesterol and the stabilization of the neuronal cytoskeleton, contributing to the preservation of synaptic integrity (3).

Humans present a polymorphism for ApoE with three alleles: $\varepsilon 2, \varepsilon 3$, and $\varepsilon 4$. Possession of allele $\varepsilon 4$ of the ApoE is the most important risk factor for the development of $\mathrm{AD}$, after advanced age $(3,6)$. The most common allele is $\varepsilon 3$, whose frequency is $60 \%$ or higher in all the populations studied. Possession of the $\varepsilon 4$ allele is associated with lower neuroplasticity and lower synaptic repair capacity, and seems to promote the relatively early appearance of brain deposits of neurotoxins, such as $A \beta$ and neurofibrillary tangles, whose excess is associated to AD.

There has been an increase in the expression of genes associated with neuronal plasticity in the human cerebral cortex, resulting in an increased capacity for learning and memory, neurotransmission, axonal transport, aerobic metabolism, and neuroprotection, all of which are adaptations that promote high neuronal activity over a long life $(6,7)$. The human brain has a high need for glucose, especially during its development. A child's brain consumes more than $40 \%$ of the body's basal energy requirements. Most of the glucose is oxidized to produce ATP. This process is upregulated in anaerobic conditions. Aerobic glycolysis is increased during childhood and is synonymous with high rates of synaptic formation and the growth and remodeling of synapses. Aerobic glycolysis is associated with the persistence of genetic expression associated with childhood, especially genes active in youth, and especially those related to the growth and formation of new synapses (transcriptional neoteny) (8). In the adult human brain, aerobic glycolysis is especially elevated in cortical areas related to cognitive functions that have undergone significant modifications during the evolution of the human species, such as the dorsolateral prefrontal cortex and the brain's default mode network (BDMN), related to the coordination of activity between different cortical areas and to planning and autobiographical memory capacities, which allow "mental travel in time," remembering and planning.

The brain regions where most of the $A \beta$ deposits are located almost exactly match the regions that make up the BDMN, which suggests that the high synaptic turnover that occurs in these areas predisposes the formation of abnormal peptide deposits characteristic of AD (8). Multiple studies show that AD appears to be associated with oxidative stress (9). Increased aerobic metabolism in neurons that retain juvenile characteristics in adulthood could subject these neurons to high 
oxidative stress. It appears that oxidative stress could induce epigenetic changes, reducing the expression of certain genes, including those related to synaptic plasticity.

\section{EPIGENETIC CHANGES AND AD}

Epigenetic changes modulate the expression of certain genes without altering the DNA sequence. Epigenetic factors include DNA methylation, histone modification, and the regulation and modification of chromatin by noncoding RNA (ncRNA) (10). DNA methylation modifies cytosine residues by adding methyl groups in regions rich in cytosine-guanine. DNA methyltransferases, such as DNA methyltransferase 1, DNA methyltransferase 2, DNA methyltransferase 3, and DNA methyltransferase 3,6, are involved in the process.

Some cytosines, for example those located in the promotor region of the APP gene, have been found to exhibit methylation with age, which can lead to the formation of $A \beta$ deposits. Methylation of the gene coding for the microtubuleassociated protein tau (MAPT) can lead to the suppression of MAPT, which can end up affecting the level of the tau protein. Further, methylation in the promotor region of the brain-derived neurotrophic factor (BDNF) gene seems to play a significant role in the appearance of mild cognitive impairment (11).

Methylation of certain loci of specific genes, such as sortilin-related receptor 1 (SORL1), ATP binding cassette subfamily A member 7 (ABCA7), HLA class II histocompatibility antigen DRB5 beta chain (HLADRB5), solute carrier family 24 member 4 (SLC24A4), and box-dependent-interacting protein 1 (BIN1), has also been associated with AD (11). The protein encoded by SORL1 controls the production of $A \beta$, so the methylation of the DNA that codifies this protein could lead to increased levels of $A \beta$.

Reelin is an extracellular matrix glycoprotein that, together with ApoE, shares the LRP and VLDLR/ApoER2 membrane receptors. During embryonic development, this protein regulates neuronal migration and, in the adult brain, intervenes in synaptic plasticity, interacting with ApoE. Binding of this protein to the membrane receptors activates a series of proteins that constitute the signaling pathway of reelin, inducing changes in the neuronal cytoskeleton. In transgenic mice that have lesions similar to those of $\mathrm{AD}$, reelin counteracts early-phase synaptic dysfunction induced by the $A \beta$ peptide (12).

In vitro studies have shown that oxidative stress alters the activation of proteins that are part of the reelin signaling pathway, resulting in the hyperphosphorylation of tau, which precedes the formation of neurofibrillary tangles in AD (13). Depletion of brain reelin has been detected in patients with $A D$ prior to the formation of $A \beta$ deposits (14). Thus, there seems to be a relationship between dysfunction of the reelin signaling pathway and $\mathrm{AD}$.

Some reelin genotypes have been found to be associated with mild cognitive impairment and AD. The reelin single nucleotide polymorphism 2299356 (RELN-rs2299356) guanine-guanine genotype is associated with cognitive decline, while the adenine-adenine genotype triples the risk of developing AD. The reelin single nucleotide polymorphism 528528 (RELN-rs528528) cytosinecytosine genotype, on the other hand, reduces the probability of mild cognitive 
impairment by two thirds. These variations are located in the promoter region of the gene, which seems to play a regulatory role in its expression (15).

Reelin is involved in neuroplasticity, a process that has increased during the evolution of the human brain. Oxidative stress and probably other factors seem to induce epigenetic changes capable of reducing the expression of genes involved in synaptic plasticity. In some cases, such as that of the carriers of certain reelin genotypes, dysfunction of the proteins involved in the reelin signaling pathway caused by a reduction in reelin related to epigenetic changes could increase the probability of having the abnormal peptide deposits that characterize AD (15). It cannot be ruled out that certain alleles are more vulnerable than others to oxidative stress, toxins, inflammation, and other factors possibly related to AD.

Histones are proteins that serve as structural support for the DNA of the cell nucleus. Nuclear DNA associates with histones to form nucleosomes. The distribution and compaction of nucleosomes determines the structure of the chromatin and the accessibility of DNA to factors involved in the transcriptional machinery. Histones are also susceptible to epigenetic changes that can cause an increase or decrease in genetic expression. Nucleosomes are mainly regulated by posttranslational modifications that occur in the N-terminal region of histones.

Both methylation and acetylation can occur in histones through the antagonistic action of histone acetyltransferases (HATs), histone deacetylases (HDACs), histone methyltransferases, and histone demethylases. The acetylation of histones results in increased genetic activity by reducing the compactness of the nucleosomes and thus facilitating access of the transcriptional machinery to DNA. During senescence, mammalian cell cultures develop highly condensed regions of chromatin that may be associated with transcriptional decline (16).

Various HDAC inhibitors, like valproic acid and sodium butyrate, seem to improve memory in animal models and some neurodegenerative diseases like Parkinson's and even AD (16). Among the epigenetic changes described are the alteration of expression of the ncRNA. ncRNA is involved in genetic silencing as well as other functions, including the regulation of the activity of retrotransposons, genes that are capable of moving from one location in the genome to another. Short fragments of ncRNA, such as microRNA (miRNA), are involved in transcriptional gene regulation. ncRNA is primarily expressed in the brain, where it is involved in neuronal development, control of regions of the genome, which are involved in neuronal migration, homeostasis, and plasticity (17).

Epigenetics has improved our understanding of the evolution of the human brain, synaptic plasticity and neuronal diversity. Several studies have identified DNA methylation, changes in histones and chromatin, and changes in ncRNA expression in various neurological diseases, including AD. A large proportion of the genes that compose our genome are expressed in the central nervous system, where a substantial amount of miRNA is also synthesized. Several factors that have been associated with $\mathrm{AD}$, such as diabetes mellitus, high blood pressure, obesity, diet, excessive sedentary lifestyle, smoking, and even a low educational level, are capable of inducing epigenetic changes (18).

There is currently no effective treatment for AD. However, cholinesterase inhibitors, such as donepezil, rivastigmine, and galantamine, together with $N$-methyl-Daspartate (NMDA) receptor antagonists, such as memantine, produce moderate and transient symptomatic benefits in the early stages of the disease. Various treatments targeting the supposed causes of the disease are being developed, all still in the 
experimental phase. One such treatment, active immunotherapy with A $\beta$ fragments, which has been effective in transgenic mice (19), has not only been clinically ineffective in human patients but has also caused encephalitis in some cases (20). Passive immunotherapy with antibodies to A $\beta$ has shown some benefits in transgenic mice and is being tested in humans. These clinical trials have shown that the clearance of $A \beta$ in humans does not appear to produce significant cognitive improvements, which has led to some researchers questioning the role that $A \beta$ plays in the cognitive decline associated with AD (20).

Attempts are also being made to develop drugs that prevent hyperphosphorylation or aggregation of the tau protein, although less effort has been made to this end than in inhibiting the formation of $A \beta$ deposits. Most researchers support the hypothesis that amyloid plaques and the neurofibrillary tangles are neurotoxic. The amyloid cascade hypothesis has led to the development of treatments that promote $A \beta$ clearance or prevent the formation of plaques. Such treatment has thus far been ineffective. A relatively high number of elderly people develop A $\beta$ deposits without presenting with cognitive decline, which calls into question the amyloid cascade hypothesis.

Recent studies show that the $A \beta$ peptide has antimicrobial properties, and that the absence of this peptide leads to an increased vulnerability to infection. Although the immune system has limited access to the central nervous system, it could fight invading pathogens with antimicrobial peptides like A $\beta$. The abnormal accumulation of $A \beta$ observed in $A D$ could be caused by persistent subacute infection or by noninfectious factors, such as trauma, ischemia, toxins, and anesthetics (21). Some researchers defend the hypothesis that $A \beta$ acts as an antioxidant in response to the oxidative stress that takes place in regions of the brain subjected to high synaptic turnover, like that which occurs in the phenomenon of neuronal neoteny, where certain neurons retain a high synaptic plasticity in adulthood.

The generation of the A $\beta$ peptide may have an adaptive function in its initial phases, and the same could be assumed about the hyperphosphorylation of tau. This would explain why drugs that reduce $A \beta$ production have not been effective so far. Attempting to reverse the epigenetic changes that occur in AD could perhaps be of therapeutic value in the future.

\section{EPIGENETIC THERAPIES IN AD}

As previously discussed, the treatments currently approved for AD are acetylcholinesterase inhibitors (donepezil, rivastigmine, and galantamine) and the glutamate NMDA receptor antagonist memantine, drugs indicated for the specific treatment of memory disorders. Acetylcholinesterase inhibitors increase the levels of the neurotransmitter acetylcholine, which is decreased in brains with AD, and NMDA receptor antagonists prevent aberrant stimulation (22). These drugs achieve a discrete and transient improvement in cognitive and functional capacities, but do not delay the progression of the disease. Nevertheless, observational studies suggest that the combination of these treatments prolongs the time until patients need to be admitted to a residence (23). As a result, there is a great deal of interest in researching new treatments for the disease. 
The main line of research in AD is that of anti-amyloid therapies (24). Despite the serious complications associated with active immunotherapy and the repeated failures of passive immunotherapy, novel anti-amyloid antibodies such as aducanumab and BAN2401 have brought fresh hope in this line of research, since they have been shown to be capable of reducing amyloid load in preliminary clinical trials (25). Other treatments within the amyloid cascade hypothesis have been developed, which promote $A \beta$ clearance or prevent plaque formation. However, not only have none of these treatments within this line of research been shown to be effective, but some of them have led to clinical worsening $(26,27)$.

Another line of research is that of anti-tau therapies, drugs that prevent the hyperphosphorylation or aggregation of the tau protein, or antibodies that reduce the levels of the protein in the cerebrospinal fluid. Lastly, other avenues of research that are currently unsuccessful or under investigation are anti-APOE4 drugs, antioxidants, anti-inflammatory drugs, cardiovascular drugs, mitochondrial protectors, hormone therapy, and antiviral drugs (28-30).

Due to the difficulty in finding effective drugs for $\mathrm{AD}$, it is crucial that other possible therapeutic avenues are explored, such as that of epigenetic drugs. This line of research is based on the fact that epigenetic changes take place during neurodevelopment and aging, and that epigenetic alterations are common in various neurodevelopmental and neurodegenerative diseases.

In the case of $\mathrm{AD}$, more than 20 epigenetic mechanisms have been identified, most of which involve direct DNA modifications (as in the case of methylation), modifications in chromatin structure (as in the case of histone modifications), or modification of mRNA-related processes, including ncRNA and miRNA.

With regard to changes in methylation in $\mathrm{AD}$, a recent study has established reference maps of the genome-wide distribution of the three possible states of DNA methylation $(5 \mathrm{mC}, 5 \mathrm{hmC}$, and $5 \mathrm{fC} / \mathrm{caC}$ ) in this disease (31). The results of this study, based mainly on cortical neurons obtained from induced pluripotent stem cells, suggest that the changes detected in DNA may precede the appearance of the disease, rather than appear later as a consequence of its progression. These results could mean these markers could be very useful in reaching early molecular diagnosis and therapy.

In addition, it has been detected in $\mathrm{AD}$ and frontotemporal dementia that the levels of an important transcriptional repressor-repressor element 1-silencing transcription factor (REST) — do not increase adequately with age (32). Consequently, transcriptional changes occur, and decreases in the expression of neuroprotector genes are found, including forkhead box protein class O (FOXO), which contributes to resistance to oxidative stress. In contrast, increased expression levels of genes that promote $\mathrm{AD}$ pathology, such as presenilin 2, are found. Taken together, these changes would increase neuronal fragility in these diseases. Furthermore, in animal models, such as the K-p25 AD mouse model, an increase in the expression of genes associated with the immune response has been detected, along with decreases in the expression of genes involved in synaptic functions and learning (33).

Several changes in the histone acetylation process, which is heavily involved in the consolidation of memory, have been detected in $\mathrm{AD}$. For example, the levels of histone $\mathrm{H} 4$ with acetylation at the 16th lysine residue protein (H4K16ac), a histone marker located in enhancers and promoters generally associated with active gene expression, are duplicated in the cerebral cortex in healthy aging but 
are barely detectable in the cerebral cortex of people with AD (34). Levels of histone deacetylase 2 (HDAC2), which increase in cultured cells after neurotoxic insults, are also found to be increased in the hippocampus and prefrontal cortex of AD mouse models and in the hippocampus of people with AD (35). Increases in deacetylase lead to worsening of synaptic function. It should be noted that blocking HDAC2 increases synaptic density and alleviates the loss of memory, but does not improve neuronal survival. This means that deficits in AD are caused not only by neuronal loss but also by epigenetic blocking of the functions of neuronal survivors (36). In addition to the reduction in expression of genes important for neuronal function, an increase in aberrant expression of genes that are normally silenced or expressed at low levels has also been observed in $\operatorname{AD}(37,38)$.

Furthermore, the expression of miRNA in the brain is altered in AD. For example, reduced levels of miRNA-29a/b-1 and miRNA-132, and increased levels of miRNA-34c have been detected. The decrease in miRNA-29a/b-1, which is a betasite amyloid precursor protein cleaving enzyme 1 (BACE1) inhibitor, correlates with an increase in the production of $A \beta$ (39). A decrease in miRNA-132, which targets the tau protein, HAT-associated protein 300 (EP300), sirtuin deacetylase 1, and FOXOla, would jeopardize neuronal growth, the integration of newborn neurons, synaptic structure, and plasticity (40). Dysregulation of miRNA expression has also been detected in biofluids, suggesting that these molecules could be used as both biomarkers and therapeutic targets $(39,40)$.

Finally, an acceleration of epigenetic age has also been observed in $\mathrm{AD}$, especially in the prefontal cortex. Epigenetic age is estimated from DNA methylation levels in $353 \mathrm{CpG}$ sites, and its acceleration with respect to chronological age is associated with, in addition to $\mathrm{AD}$, higher mortality, cognitive impairment, and other neurodegenerative diseases $(41,42)$. Epigenetic age could also explain differences in the onset age of $\mathrm{AD}$ in members of the same family that share the same gene mutation (43). That is, those who have an accelerated epigenetic age would develop $\mathrm{AD}$ symptoms at an earlier age.

The goal of epigenetic therapies is to reverse at least some of the epigenetic changes caused by AD. Such therapies have several advantages. First, specific drugs can be designed because the epigenetic changes are induced by enzymes that act at the DNA or histone level. Second, they act on reversible mechanisms since the epigenetic changes at the DNA and histone level are both regulated by enzymes. Finally, these therapies enable us to unite physiology and pathology, because epigenetics influence gene expression throughout life, and thus epigenetic drugs would be effective in both neurodevelopmental and neurodegenerative diseases. In addition, epigenetic therapies can target any component of the epigenetic machinery.

In the last decade, several epigenetic drugs have been designed for the treatment of neurological diseases. The most promising are DNA-demethylating agents and HDAC inhibitors (HDACis). In fact, there are already drugs of these two therapeutic groups that have been approved by the US Food and Drug Administration for the treatment of hematological cancer. In the former group, there is 5-azactydine and the 5-aza-2'-deoxycytidine (or decitabine), and in the latter group, suberoylanilide hydroxamic acid (SAHA or vorinostat), romidepsin, belinostat, panobinostat, and chidamide have been approved.

HDAC aims to regulate imbalances in protein acetylation levels and transcription. Their use in neurodegenerative diseases is based on their neuroprotective, 
neurotrophic, and anti-inflammatory properties. In the case of AD, HDACs play an important role in memory consolidation and could be useful as therapeutic targets. For example, HDAC2 and HDAC3 have been shown to play a repressive role in memory formation, while HDAC5 has a memory-enhancing effect (44). As a result, HDAC2-inhibiting drugs, such as CI-994, and HDAC5-enhancing drugs could be useful in AD. The therapeutic potential of HDACis has been demonstrated in studies with animals. In APP/PS1 mice, acute treatment with HADCi trichostatin A (TSA), sodium valproic acid, SAHA, sodium butyrate (NaB), butyrate, vorinostat, 4-phenylbutiric acid, MS-275, and crebinostat improved the cognitive performance of these animals (45-49).

Sulforaphane could also be useful in AD. Sulforaphane is an HDACi that decreased HDAC2 levels in the triple-transgenic mouse model of $A D(3 \times T$ T-AD). This was accompanied by an increase in the acetylation of histones $\mathrm{H} 3$ and $\mathrm{H} 4$ in the BDNF promoter and resulting in an increase in its expression (50).

There are HDACis that affect multiple genes involved in $\mathrm{AD}$, which could be advantageous given the multifactorial etiology of $\mathrm{AD}$. The disadvantage of these compounds is that their wide spectrum theoretically broadens the possibilities of adverse effects with their use. This group includes M344 \{4-(dimethylamino)-n[7-(hydroxyamino)-7-oxoheptyl]benzamide\}, CM-414 \{3-[[4-ethoxy-3-(1-methyl7-oxo-3-propyl-6H-pyrazolo [4,3-d] pyrimidin-5-yl)phenyl] methyl]N-hydroxycyclobutane-1-carboxamide\}, and RGFP-966 \{(E)-N-(2-amino-4fluorophenyl)-3-[1-[(E)-3-phenylprop-2-enyl]pyrazol-4-yl]prop-2-enamide\}. Their chronic use in animal models showed cognitive benefits (51-57).

In addition to the HDACis described, there are other HDACis that have been specifically designed. In this way, HDACi W2 was obtained, which features a longer half-life and better penetration of the blood-brain barrier than the HDACis currently available. HDACi W2 has been shown to be capable of significantly reducing $A \beta$ levels in hAPP $3 \times \operatorname{Tg} A D$ mice by reducing the expression of genes involved in $A \beta$ production and increasing the expression of $A \beta$ degradation enzymes. This HDACi is also capable of decreasing phosphorylation of the tau protein, promoting the formation and growth of dendritic spines, and improving learning and memory in these mice, which makes it a potential candidate for the treatment of $\mathrm{AD}$.

Due to the observed benefits of using HDACis in animal models of $A D$, there are several ongoing clinical studies with these compounds. One of the compounds under study is valproate, which is a class I HDAC inhibitor. It has already been approved for treating epilepsy, migraine, and bipolar disorder, and since its activity as a HDACi was discovered, it is also being studied to evaluate its effectiveness in neurodegenerative disease. In preclinical studies, it was shown to be effective in reversing cognitive impairment in a mouse model of $\operatorname{AD}(58,59)$.

Another drug under study is vitamin B3 or nicotamide, which is also a class III NAD-dependent sirtuin HDAC inhibitor. This compound can delay aging in mouse oocytes and delay cognitive impairment in a mouse model of AD (60). A phase I clinical trial in patients with $\mathrm{AD}$ demonstrated its safety. It is currently in a phase II clinical trial. Another HDACi, vorinostat, is being evaluated in a phase I clinical trial in patients with AD. Finally, RDN-929, which is a CoRESTselective HDAC inhibitor that could reactivate neuronal gene expression, strengthen synaptic function, and promote new synapses, has been studied in two phase I clinical trials as a possible treatment for AD. 
Another mechanism for enhancing acetylation is the use of drugs that enhance HATs. The increased expression of an enzyme of this type, Tip60, in drosophila overexpressing human APP was able to restore the benefits of environmental enrichment (61).

With regard to miRNA, utility of the drug gemfibrozil has been studied. Gemfibrozil is capable of modifying miR-107 levels, the reduction of which may accelerate the progression of AD by regulating the expression of BACE1. A phase I clinical trial showed the drug was safe and reduced miR-107 in plasma and in CSF to undetectable levels.

Other possible epigenetic therapeutic targets not yet explored in AD are drugs directed against HATs, ten-eleven-translocation methylcitosine-dioxygenases enzymes (which catalyze the conversion of 5-methylcytosine to 5-hydroxymethylcytosine), DNA demethylation, chromatine remodelers, and other histone modifications.

Finally, it should be noted that there are also nonspecific epigenetic therapies that can be useful in AD. The first is blood plasma therapy from young subjects. In a recent study, in which aged mice were treated with blood plasma from young mice, it was observed that the treatment halved the epigenetic ages of blood, heart, and liver tissue, and also rejuvenated the hypothalamus. The treatment also improved the functioning of these organs as well as cognitive functions (62). The second nonspecific epigenetic therapy is cognitive stimulation, which has been shown to be capable of causing epigenetic changes (63). The third nonspecific epigenetic therapy is physical exercise. In animals, physical exercise is capable of reversing age-related reduction of adult neurogenesis and cognitive function in the aged hippocampus (64). In addition, a recent study showed that the administration of circulating blood factors in the plasma of aged mice subjected to exercise was capable of transferring the beneficial effects to sedentary-aged mice. These investigations led to the discovery that glycosylphosphatidylinositol (GPI)-specific phospholipase Dl (Gpld1), a GPIdegrading enzyme derived from liver, was probably responsible for these effects.

Although epigenetic therapies have a promising future role in AD, there are still problems that must first be addressed. First, we need to bear in mind that perhaps not all epigenetic changes can be reversed with these types of therapy. Second, epigenetic changes are extremely complex, and the therapies could have any number of side effects that are difficult to control. Third, different regions of the same gene can have antagonistic epigenetic changes, so the effect of an epigenetic therapy could be unpredictable. With respect to pharmacological properties, current therapies lack specificity and are not selective for specific brain regions, cell types, or genes. This limitation could be addressed with the use of siRNA or the use of chromatin-modifying enzymes, transcription activation-like effectors or clustered regularly interspaced short palindromic repeats/ Cas System, and the use of artificial transcriptional factors of the silencing or promoter type.

The development of new study techniques will also be essential to better understand how epigenetic therapies work and to be able to design future drugs. First, laboratory techniques such as cell-type specific analysis of transcription and DNA methylation in the brain, single cell analysis of DNA-protein interactions, and chromatin 3D structure might be applied in future studies to uncover neuronal cell-type specific chromatin structure and interneuronal variations. Furthermore, 
new models for studying the effect of these therapies, such as neuronal cultures or other brain cell types derived from human stem cells or induced pluripotent stem cells, could be better than the animal models used so far. The application of innovative imaging techniques, such as positron emission tomography using radiochemical $\left[{ }^{11} \mathrm{C}\right]$ martinostat, which binds specifically to certain HDAC isoforms, could help to discover the gene expression patterns regulated by chromatinmodifying enzymes in the live brain. Finally, establishing epigenetic biomarkers for diagnosis, prognosis, and therapy would be important to test the efficacy of epigenetic drugs and to classify patients according to the particular therapy they would most benefit from.

\section{CONCLUSION}

At present, there are no treatments for AD. Given that several epigenetic changes occur in this disease, treatments aimed at reversing these changes may constitute promising lines of research in the future.

Conflict of Interest: The authors declare no potential conflicts of interest with respect to research, authorship, and/or publication of this chapter.

Copyright and Permission Statement: To the best of our knowledge, the materials included in this chapter do not violate copyright laws. All original sources have been appropriately acknowledged and/or referenced. Where relevant, appropriate permissions have been obtained from the original copyright holder(s).

\section{REFERENCES}

1. Ballard C, Gauthier S, Corbett A, Brayne C, Aarscland D, Jones E. Alzheimer's disease. Lancet. 2011 Mar 19;377(9770):109-31. http://dx.doi.org/10.1016/S0140-6736(10)61349-9

2. Weiner MF, Lipton AM, editors. Enfermedad de Alzheimer. Manual de enfermedad de Alzheimer y otras demencias. Madrid: Editorial Médica Panamericana; 2009. p. 155-72.

3. Mesulam MM. Principles of behavioral and cognitive neurology. 2nd ed. New York: Oxford University Press; 2000.

4. Niu H, Alvarez Alvarez I, Guillen Grima F, Arguinaga Ontosa I. Prevalence and incidence of Alzheimer's disease in Europe: A meta-analysis. Neurologia. 2017;32(8):525-532. Spanish. http:// dx.doi.org/10.1016/j.nrleng.2016.02.009

5. Braak H, Rüb U, Schultz C, del Tredici K. Vulnerability of cortical neurons to Alzheimer's and Parkinson diseases. J Alzheimers Dis. 2006;9:35-44. http://dx.doi.org/10.3233/JAD-2006-9S305

6. Cáceres M, Lachuer J, Zapala M, Redmond J, Kado L, Geshwind D, et al. Elevated gene expression levels distinguish human from non-human primate brains. Proc Natl Acad Sci U S A. 2003;100:13030-5. http://dx.doi.org/10.1073/pnas.2135499100

7. Uddin M, Wildman DE, Liu G, Xu W, Johnson RM, Patrick RH, et al. Sister grouping of chimpanzees and humans as revealed by genome-wide phylogenetic analysis of brain gene expression profiles. Proc Natl Acad Sci U S A. 2004;101(9):2957-62. http://dx.doi.org/10.1073/pnas.0308725100

8. Bufill E, Agusti J, Blesa R. Human neoteny revisited: The case of synaptic plasticity. Am J Hum Biol. 2011;23:729-39. http://dx.doi.org/10.1002/ajhb.21225 
9. Zawia NH, Lahiri DK, Cardozo-Pelaez E. Epigenetics, oxidative stress and Alzheimer disease. Free Radic Biol Med. 2009;46:1241-9. http://dx.doi.org/10.1016/j.freeradbiomed.2009.02.006

10. Liu X, Jiao B, Shen L. The epigenetics of Alzheimer disease: Factors and therapeutic implications. Front Genet. 2018 Nov 30;9:579. http://dx.doi.org/10.3389/fgene.2018.00579

11. Traynor EJ, Renton AE. Exploring the epigenetics of Alzheimer's disease. JAMA Neurol. 2015;72(1):8-9. http://dx.doi.org/10.1001/jamaneurol.2014.3057

12. Rogers JT, Rusiana I, Trotter J, Zhao L, Donaldson E, Pak DTS, et al. Reelin supplementation enhances cognitive ability, synaptic plasticity and dendritic spine density. Learn Mem. 2011 Sep;18(9):558-64. http://dx.doi.org/10.1101/lm.2153511

13. Patrick GN, Zukerberg L, Nikolic M, de la Monte S, Dikkes P, Tsai LH. Conversion of p35 to p25, deregulates Cdk5 and promotes neurodegeneration. Nature. 1999 Dec;9(402):615-22. http://dx.doi. org/10.1038/45159

14. Stranahan AM, Haberman RP, Gallagher M. Cognitive decline is associated with reduced reelin expression in the entorhinal cortex of aged rats. Cereb Cortex. 2011 Feb;21(2):392-400. http://dx.doi. org/10.1093/cercor/bhq106

15. Bufill E, Roura-Poch P, Sala-Matavera I, Anton S, Lleó A, Sanchez Saudinós B, et al. Reelin signaling pathway genotypes and Alzheimer disease in a Spanish population. Alzheimer Dis Assoc Disord. 2015 April-June;29(2):169-72.

16. Sanchez-Mut JV, Gräff J. Epigenetic alterations in Alzheimer's disease. Front Behav Neurosci. 2015 Dec 17;9:347. http://dx.doi.org/10.3389/fnbeh.2015.00347

17. Qureshi IA, Mark F, Mehler MD. Understanding neurological disease mechanisms in the era of epigenetics. JAMA Neurol. 2013;70(6):703-10. http://dx.doi.org/10.1001/jamaneurol.2013.1443

18. Sen P, Shah P, Nativio R, Berger SL. Epigenetic mechanisms for longevity and aging. Cell. 2016 Aug 11;166:822-39. http://dx.doi.org/10.1016/j.cell.2016.07.050

19. Schenk D, Barbour R, Dunn W, Gordon G, Grajeda H, Guido T, et al. Immunization with amyloid- $\beta$ attenuates Alzheimer disease like pathology in the PDAPP mouse. Nature. 1999;400:173-7. http:// dx.doi.org/10.1038/22124

20. Arai H. [Immunotherapy and Alzheimer's disease]. Nihon Shinkei Seishin Yakurigaku Zasshi. 2010 Aug;30(4):173-6. Japanese.

21. Soscia SJ, Kirby JE, Washicosky KJ, Tucker SM, Ingelsson M, Hyman B, et al. The Alzheimer's disease-associated amyloid beta-protein is an antimicrobial peptide. PLoS One. 2010 Mar 3;5(3):e9505. http://dx.doi.org/10.1371/journal.pone.0009505

22. Cummings JL. Alzheimer's disease. N Engl J Med. 2004;351(1):56-67. http://dx.doi.org/10.1056/ NEJMra040223

23. Lopez OL, Becker JT, Wahed AS, Saxton J, Sweet RA, Wolk DA, et al. Long-term effects of the concomitant use of memantine with cholinesterase inhibition in Alzheimer disease. J Neurol Neurosurg Psychiatry. 2009 Jun;80(6):600-7. http://dx.doi.org/10.1136/jnnp.2008.158964

24. Cummings J, Lee G, Ritter A, Sabbagh M, Zhong K. Alzheimer's disease drug development pipeline: 2020. Alzheimer's Dement (NY). 2020 Jul 16;6(1):e12050. http://dx.doi.org/10.1002/trc2.12050

25. Logovinsky V, Satlin A, Lai R, Swanson C, Kaplow J, Osswald G, et al. Safety and tolerability of BAN2401-A clinical study in Alzheimer's disease with a protofibril selective A $\beta$ antibody. Alzheimers Res Ther. 2016 Apr 6;8(1):14. http://dx.doi.org/10.1186/s13195-016-0181-2

26. Egan MF, Kost J, Voss T, Mukai Y, Aisen PS, Cummings JL, et al. Randomized trial of verubecestat for prodromal Alzheimer's disease. N Engl J Med. 2019;380:1408-20. http://dx.doi.org/10.1056/ NEJMoal 812840

27. Novak G, Streffer JR, Timmers M, Henley D, Brashear HR, Bogert J, et al. Long-term safety and tolerability of atabecestat (JNJ-54861911), an oral BACEl inhibitor, in early Alzheimer's disease spectrum patients: A randomized, double-blind, placebo-controlled study and a two-period extension study. Alzheimers Res Ther. 2020 May;12(1):58. http://dx.doi.org/10.1186/s13195-020-00614-5

28. Lee HP, Zhu X, Casadesus G, Castellani RJ, Nunomura A, Smith MA, et al. Antioxidant approaches for the treatment of Alzheimer's disease. Expert Rev Neurother. 2010 Jul;10(7):1201-8. http://dx.doi. org/10.1586/ern.10.74

29. Cole GM, Frautschy SA. Mechanisms of action of non-steroidal anti-inflammatory drugs for the prevention of Alzheimer's disease. CNS Neurol Disord Drug Targets. 2010 Apr;9(2):140-8. http://dx.doi. org/10.2174/187152710791011991 
30. Bezprozvanny I. The rise and fall of Dimebon. Drug News Perspect. 2010 Oct;23(8):518-23. http:// dx.doi.org/10.1358/dnp.2010.23.8.1500435

31. Fetahu IS, Ma D, Rabidou K, Argueta C, Smith M, Liu H, et al. Epigenetic signatures of methylated DNA cytosine in Alzheimer's disease. Sci Adv. 2019 Aug 28;5(8):eaaw2880. http://dx.doi. org/10.1126/sciadv.aaw2880

32. Lu T, Aron L, Zullo J, Pan Y, Kim H, Chen Y, et al. REST and stress resistance in ageing and Alzheimer's disease. Nature. 2014 Mar 27;507(7493):448-54. http://dx.doi.org/10.1038/nature13163

33. Gjoneska E, Pfenning AR, Mathys H, Quon G, Kundaje A, Tsai L-H, et al. Conserved epigenomic signals in mice and humans reveal immune basis of Alzheimer's disease. Nature. 2015 Feb;518(7539):365-9. http://dx.doi.org/10.1038/nature14252

34. Nativio R, Donahue G, Berson A, Lan Y, Amlie-Wolf A, Tuzer F, et al. Dysregulation of the epigenetic landscape of normal aging in Alzheimer's disease. Nat Neurosci. 2018;21(4):497-505. http://dx.doi. org/10.1038/s41593-018-0101-9

35. Gräff J, Rei D, Guan J-S, Wang W-Y, Seo J, Hennig KM, et al. An epigenetic blockade of cognitive functions in the neurodegenerating brain. Nature. 2012 Feb 29;483(7388):222-6. http://dx.doi. org/10.1038/nature 10849

36. Liu D, Tang H, Li XY, Deng MF, Wei N, Wang X, et al. Targeting the HDAC2/HNF-4A/miR-101b/ AMPK pathway rescues tauopathy and dendritic abnormalities in Alzheimer's disease. Mol Ther. 2017 Mar 1;25(3):752-64. http://dx.doi.org/10.1016/j.ymthe.2017.01.018

37. Frost B, Hemberg M, Lewis J, Feany MB. Tau promotes neurodegeneration through global chromatin relaxation. Nat Neurosci. 2014 Mar;17(3):357-66. http://dx.doi.org/10.1038/nn.3639

38. Sugeno N, Jäckel S, Voigt A, Wassouf Z, Schulze-Hentrich J, Kahle PJ. $\alpha$-Synuclein enhances histone H3 lysine-9 dimethylation and H3K9me2-dependent transcriptional responses. Sci Rep. 2016 Nov 3;6:36328. http://dx.doi.org/10.1038/srep36328

39. Hébert SS, Horré K, Nicolaï L, Papadopoulou AS, Mandemakers W, Silahtaroglu AN, et al. Loss of microRNA cluster miR-29a/b-1 in sporadic Alzheimer's disease correlates with increased BACEl/ beta-secretase expression. Proc Natl Acad Sci U S A. 2008 Apr 29;105(17):6415-20. http://dx.doi. org/10.1073/pnas.0710263105

40. Grasso M, Piscopo P, Confaloni A, Denti MA. Circulating miRNAs as biomarkers for neurodegenerative disorders. Molecules. 2014 May 23;19(5):6891-910. http://dx.doi.org/10.3390/molecules19056891

41. Denk J, Boelmans K, Siegismund C, Lassner D, Arlt S, Jahn H. MicroRNA profiling of CSF reveals potential biomarkers to detect Alzheimer's disease. PLoS One. 2015 May;10(5):e0126423. http:// dx.doi.org/10.1371/journal.pone.0126423

42. Marioni RE, Shah S, McRae AF, Chen BH, Colicino E, Harris SE, et al. DNA methylation age of blood predicts all-cause mortality in later life. Genome Biol. 2015 Jan 30;16(1):25. http://dx.doi. org/10.1186/s13059-015-0584-6

43. Marioni RE, Shah S, McRae AF, Ritchie SJ, Muniz-Terrera G, Harris SE, et al. The epigenetic clock is correlated with physical and cognitive fitness in the Lothian Birth Cohort 1936. Int J Epidemiol. 2015 Aug;44(4):1388-96. http://dx.doi.org/10.1093/ije/dyu277

44. Horvath S, Erhart W, Brosch M, Ammerpohl O, von Schönfels W, Ahrens M, et al. Obesity accelerates epigenetic aging of human liver. Proc Natl Acad Sci U S A. 2014 Oct 28;111(43):15538-43. http:// dx.doi.org/10.1073/pnas.1412759111

45. Yang SS, Zhang R, Wang G, Zhang YF. The development prospection of HDAC inhibitors as a potential therapeutic direction in Alzheimer's disease. Transl Neurodegener. 2017 Jul 10;6:19. http://dx.doi. org/10.1186/s40035-017-0089-1

46. Göttlicher M, Minucci S, Zhu P, Krämer OH, Schimpf A, Giavara S, et al. Valproic acid defines a novel class of HDAC inhibitors inducing differentiation of transformed cells. EMBO J. 2001 Dec 17;20(24):6969-78. http://dx.doi.org/10.1093/emboj/20.24.6969

47. Morris MJ, Mahgoub M, Na ES, Pranav H, Monteggia LM. Loss of histone deacetylase 2 improves working memory and accelerates extinction learning. J Neurosci. 2013 Apr 10;33(15):6401-11. http://dx.doi.org/10.1523/JNEUROSCI.1001-12.2013

48. McQuown SC, Barrett RM, Matheos DP, Post RJ, Rogge GA, Alenghat T, et al. HDAC3 is a critical negative regulator of long-term memory formation. J Neurosci. 2011 Jan 12;31(2):764-74. http://dx.doi. org/10.1523/JNEUROSCI.5052-10.2011 
49. Rogge GA, Singh H, Dang R, Wood MA. HDAC3 is a negative regulator of cocaine-context-associated memory formation. J Neurosci. 2013 Apr 10;33(15):6623-32. http://dx.doi.org/10.1523/ JNEUROSCI.4472-12.2013

50. Kim J, Lee S, Choi BR, Yang H, Hwang Y, Park JH, et al. Sulforaphane epigenetically enhances neuronal BDNF expression and TrkB signaling pathways. Mol Nutr Food Res. 2017 Feb;61(2). http:// dx.doi.org/10.1002/mnfr.201600194

51. Francis YI, Fà M, Ashraf H, Zhang H, Staniszewski A, Latchman DS, et al. Dysregulation of histone acetylation in the APP/PS1 mouse model of Alzheimer's disease. J Alzheimer's Dis. 2009;18:131-9. http://dx.doi.org/10.3233/JAD-2009-1134

52. Kilgore M, Miller CA, Fass DM, Hennig KM, Haggarty SJ, Sweatt JD, et al. Inhibitors of class 1 histone deacetylases reverse contextual memory deficits in a mouse model of Alzheimer's disease. Neuropsychopharmacology. 2010 Mar;35(4):870-80. http://dx.doi.org/10.1038/npp.2009.197

53. Fischer A, Sananbenesi F, Wang X, Dobbin M, Tsai L-H. Recovery of learning and memory is associated with chromatin remodelling. Nature. 2007;447(7141):178-82. http://dx.doi.org/10.1038/ nature 05772

54. Rustenhoven J, Smith AM, Smyth LC, Jansson D, Scotter EL, Swanson MEV, et al. PU.1 regulates Alzheimer's disease-associated genes in primary human microglia. Mol Neurodegener. 2018 Aug 20;13(1):44. http://dx.doi.org/10.1186/s13024-018-0277-1

55. Volmar CH, Salah-Uddin H, Janczura KJ, Halley P, Lambert G, Wodrich A, et al. M344 promotes nonamyloidogenic amyloid precursor protein processing while normalizing Alzheimer's disease genes and improving memory. Proc Natl Acad Sci U S A. 2017 Oct 24;114(43):E9135-44. http://dx.doi. org/10.1073/pnas.1707544114

56. Cuadrado-Tejedor M, Garcia-Barroso C, Sánchez-Arias JA, Rabal O, Pérez-González M, Mederos S, et al. A first-in-class small-molecule that acts as a dual inhibitor of HDAC and PDE5 and that rescues hippocampal synaptic impairment in Alzheimer's disease mice. Neuropsychopharmacology. 2017 Jan;42(2):524-39. http://dx.doi.org/10.1038/npp.2016.163

57. Janczura KJ, Volmar C-H, Sartor GC, Rao SJ, Ricciardi NR, Lambert G, et al. Inhibition of HDAC3 reverses Alzheimer's disease-related pathologies in vitro and in the 3xTg-AD mouse model. Proc Natl Acad Sci U S A. 2018 Nov 20;115(47):El1148-57. http://dx.doi.org/10.1073/pnas.1805436115

58. Qing H, He G, Ly PT, Fox CJ, Staufenbiel M, Cai F, et al. Valproic acid inhibits Abeta production, neuritic plaque formation, and behavioral deficits in Alzheimer's disease mouse models. J Exp Med. 2008 Nov 24;205(12):2781-9. http://dx.doi.org/10.1084/jem.20081588

59. Yao Z, Liang L, Liu Y, Zhang L, Zhu H, Huang L, et al. Valproate improves memory deficits in an Alzheimer's disease mouse model: Investigation of possible mechanisms of action. Cell Mol Neurobiol. 2014;34(6):805-12. http://dx.doi.org/10.1007/s10571-013-0012-y

60. Green KN, Steffan JS, Martinez-Coria H, Sun X, Schreiber SS, Thompson LM, et al. Nicotinamide restores cognition in Alzheimer's disease transgenic mice via a mechanism involving sirtuin inhibition and selective reduction of Thr231-phosphotau. J Neurosci. 2008 Nov 5;28(45):11500-10. http:// dx.doi.org/10.1523/JNEUROSCI.3203-08.2008

61. Xu S, Panikker P, Iqbal S, Elefant F. Tip60 HAT action mediates environmental enrichment induced cognitive restoration. PLoS One. 2016 Jul 25;11(7):e0159623. http://dx.doi.org/10.1371/journal. pone.0159623

62. Villeda S, Plambeck K, Middeldorp J, Castellano JM, Mosher KI, Luo J, et al. Young blood reverses agerelated impairments in cognitive function and synaptic plasticity in mice. Nat Med. 2014;20:659-63. http://dx.doi.org/10.1038/nm.3569

63. Pulkett R, Lubin FD. Epigenetic mechanisms in experience-driven memory formation and behavior. Epigenomics. 20110ct;3(5):649-64. http://dx.doi.org/10.2217/epi.11.86

64. Horowitz AM, Fan X, Bieri G, Smith LK, Sánchez-Díaz CI, Schroer AB, et al. Blood factors transfer beneficial effects of exercise on neurogenesis and cognition to the aged brain. Science. $2020 \mathrm{Jul}$ 10;369(6500):167-73. http://dx.doi.org/10.1126/science.aaw2622 